\title{
Penetration and radial force balance in field-reversed configuration with large rotating magnetic field
}

\author{
M. Ohnishi, ${ }^{1}$ W. Hugrass, ${ }^{2}$ M. Fukuhara, ${ }^{1}$ T. Masaki, ${ }^{1}$ H. Osawa, ${ }^{1}$ and T. Chikano ${ }^{1}$ \\ ${ }^{1}$ Department of Electrical Engineering and Computer Science, Kansai University, 3-3-35 Yamate-cho, \\ Suita-shi, Osaka 564-8680, Japan \\ ${ }^{2}$ School of Computing, University of Tasmania, Locked Bag 1359, Launceston, Tasmania 7250, Australia
}

(Received 13 June 2008; accepted 4 September 2008; published online 15 October 2008; publisher error corrected 17 October 2008)

\begin{abstract}
A field-reversed configuration (FRC) is formed by applying a rotating magnetic field (RMF) much larger than the axial magnetic field to a cylindrical glass vacuum chamber filled with $10 \mathrm{~Pa}$ argon gas without a preionization. The FRC with the plasma density $2.2 \times 10^{19} \mathrm{~m}^{-3}$, the temperature $8.0 \mathrm{eV}$, the separatrix length $0.45 \mathrm{~m}$, and the separatrix radius $0.035 \mathrm{~m}$ is sustained for the notably long period of $40 \mathrm{~ms}$. It is observed that the antenna current which produces the RMF is reduced by about half after the FRC is formed. The interaction between the plasma and the antenna circuit increases the antenna resistance and changes the inductance of the antenna so that the circuit becomes nonresonant. The RMF is sufficiently large to fully penetrate to the center during the period and drive the current with a rigid rotor profile. The RMF is shown to play a major role in sustaining the plasma pressure. (C) 2008 American Institute of Physics. [DOI: 10.1063/1.2997341]
\end{abstract}

A rotating magnetic field (RMF) has been used for the purpose of the slow formation and the sustainment of a fieldreversed configuration (FRC). When the RMF frequency satisfies the conditions $\omega_{c i} \leqslant \omega \ll \omega_{c e}$ and $\nu_{e i} \ll \omega_{c e}$ (Ref. 1), where $\omega_{c i}$ and $\omega_{c e}$ are the cyclotron frequency of ions and electrons for RMF and $\nu_{e i}$ the electron-ion collision frequency, the RMF drives plasma current and forms FRCs with temperature about $10-100 \mathrm{eV}$, and density about $10^{18}$ $-10^{19} \mathrm{~m}^{-3} \cdot{ }^{2-7}$ The conditions for the RMF to completely penetrate into the plasma and drive the current were obtained empirically from numerical simulations, ${ }^{8,9}$

$$
\omega_{c e} / \nu_{e i}>1.12 R / \delta \text { for } R / \delta \leqslant 6.5
$$

and

$$
\begin{aligned}
\omega_{c e} / \nu_{e i}> & 1.12 R / \delta\left[1.0+0.12(R / \delta-6.5)^{0.4}\right] \\
& \text { for } R / \delta>6.5
\end{aligned}
$$

where $R$ the separatrix radius and $\delta=\left(2 \eta / \mu_{0} \omega\right)^{1 / 2}$ is the classical skin depth. The magnitude of the RMF required to penetrate into the plasma does not depend on the axial field.

The effect of the RMF on the pressure balance equations was assumed negligible in the early theoretical investigations ${ }^{10,11}$ and this assumption was verified by detailed measurements of the magnetic field, plasma density, and temperature. ${ }^{12}$ In a more recent work, Steinhauer ${ }^{13}$ showed that there is a correction RMF term in the pressure balance equations. We report here the first experimental evidence for the effect of the RMF on the radial pressure balance in an FRC sustained by an RMF.

Figure 1 shows a schematic diagram of the experimental device. The cylindrical vacuum chamber is made of Pyrex glass and has an internal radius of $35 \mathrm{~mm}$. The length of the discharge chamber is $600 \mathrm{~mm}$. The vacuum is achieved to be less than $10^{-4} \mathrm{~Pa}$ in $10 \mathrm{~min}$ by a rotary pump and a turbomolecular pump in a series. Argon gas is fed from the opposite side to the vacuum pumping system. The axial field is produced by six coils and the strength is fairly uniform along the central axis and $60 \mathrm{G}$ at the maximum. Two RMF power supplies, of which sinusoidal output is $90^{\circ}$ out of phase, deliver the current $300 \mathrm{~A}$ with the frequency $200 \mathrm{kHz}$ and output power $26 \mathrm{~kW}$ for $40 \mathrm{~ms}$. The current decreases to $200 \mathrm{~A}$ at $t=40 \mathrm{~ms}$ due to the limited capacity of the condenser. The two turn antennas are placed directly on the outer wall of the chamber in order to produce the largest RMF inside the chamber. The amplitude of the RMF in the vacuum is initially $120 \mathrm{G}$ at the center and reduces to $80 \mathrm{G}$ at $t=40 \mathrm{~ms}$ due to the decrease of the antenna current. The production of current in the antenna uses an R-L-C series resonant circuit and insulated gate bipolar transistor semiconductor switching devices. The amplitude of the RMF is large enough for full penetration during the discharge.

There are three ports in the chamber, into which the

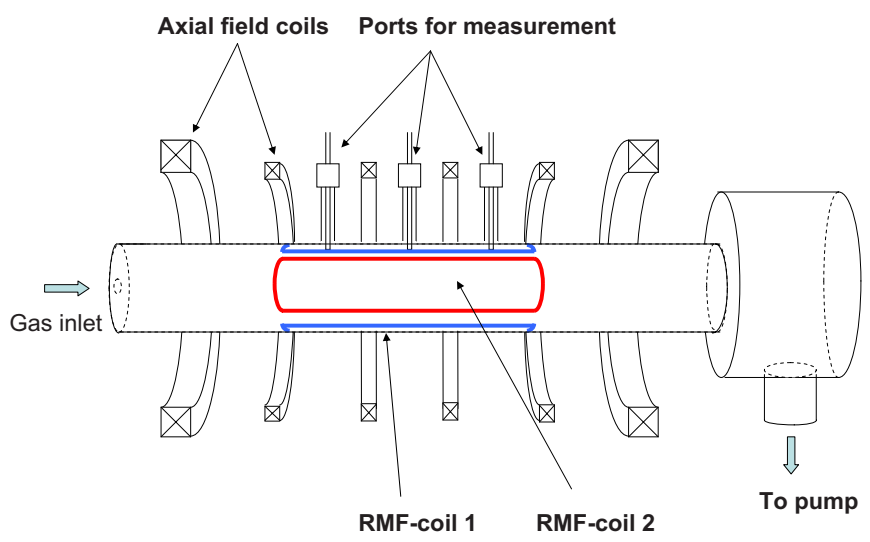

FIG. 1. (Color online) Schematic diagram of experimental device. 


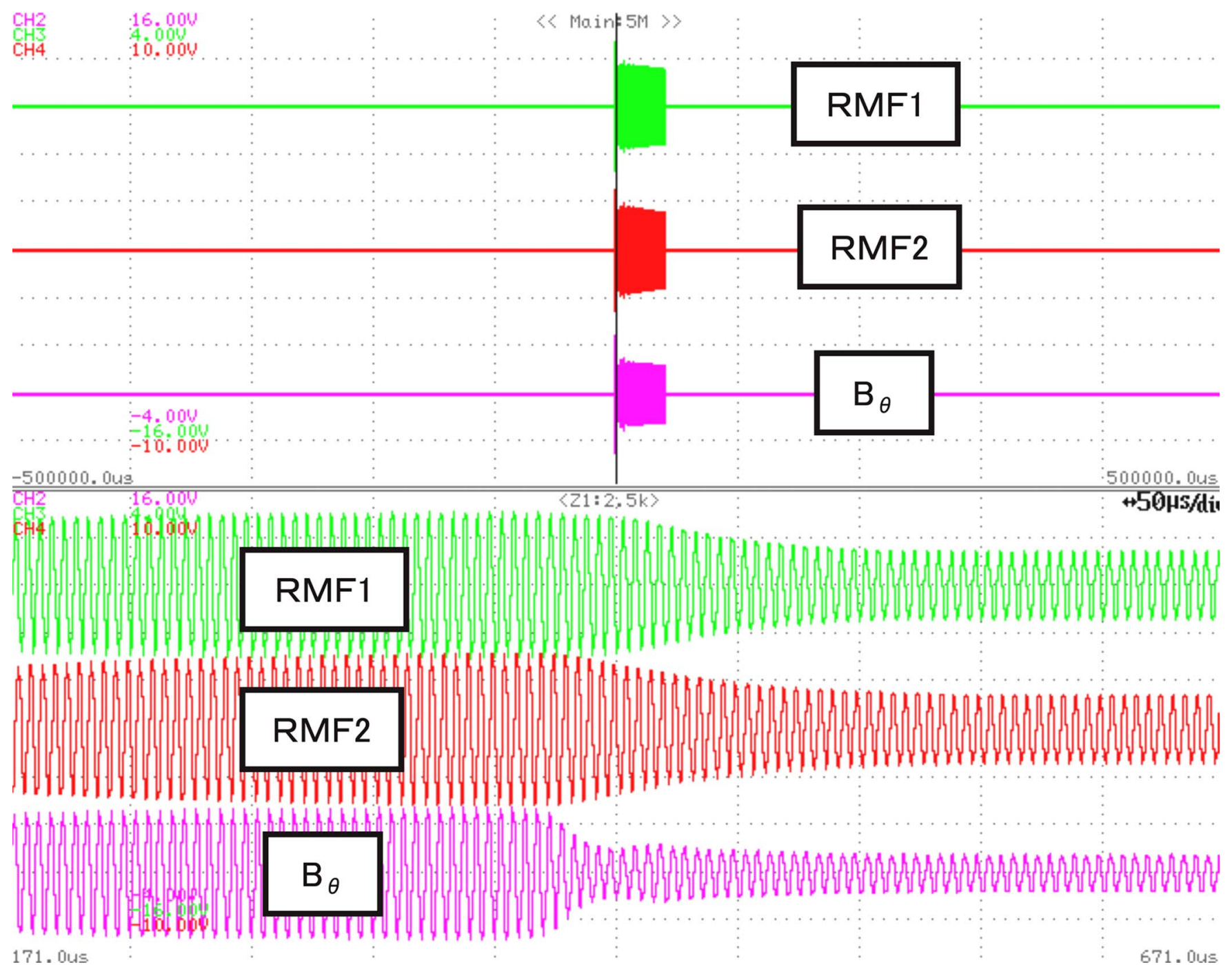

FIG. 2. (Color online) Antenna currents and the $\theta$ component of RMF in the center of vacuum chamber. Lower three curves are the enlargements of them about the instance of the plasma formation.

magnetic and electrostatic probes are inserted. The former probe is mounted on an optical rotational stage so that its orientation can be precisely adjusted in order to eliminate any pick up from the RMF when we measure the axial magnetic field, while the probe can be rotated by $90^{\circ}$ in order to measure the $\theta$ component of the RMF. The latter probe gives us the plasma density and the electron temperature. The probes are movable in the radial direction to measure the radial profiles. Eight 25-turn coils are wound around the chamber to measure the excluded magnetic flux so that we can estimate the length of the separatrix.

The axial field rises to reach $25 \mathrm{G}$ in $5 \mathrm{~ms}$. When applying the RMF, the axial field reduces to about $-27 \mathrm{G}$ in $4 \mathrm{~ms}$ at the center $(r=0, z=0)$ to $20 \mathrm{G}$ near the wall $(r=35 \mathrm{~mm}$, $z=0$ ). Figure 2 shows the time behaviors of antenna currents and the $\theta$ component of the RMF at the instance of the plasma production. The antenna current is initially $300 \mathrm{~A}$ and reduced to $155 \mathrm{~A}$ after a plasma is formed. The $\theta$ component of the RMF $120 \mathrm{G}$ reduces to $35 \mathrm{G}$ due to the decrease of the antenna currents and the induction of a shielding current, but still exists in the center, which shows the

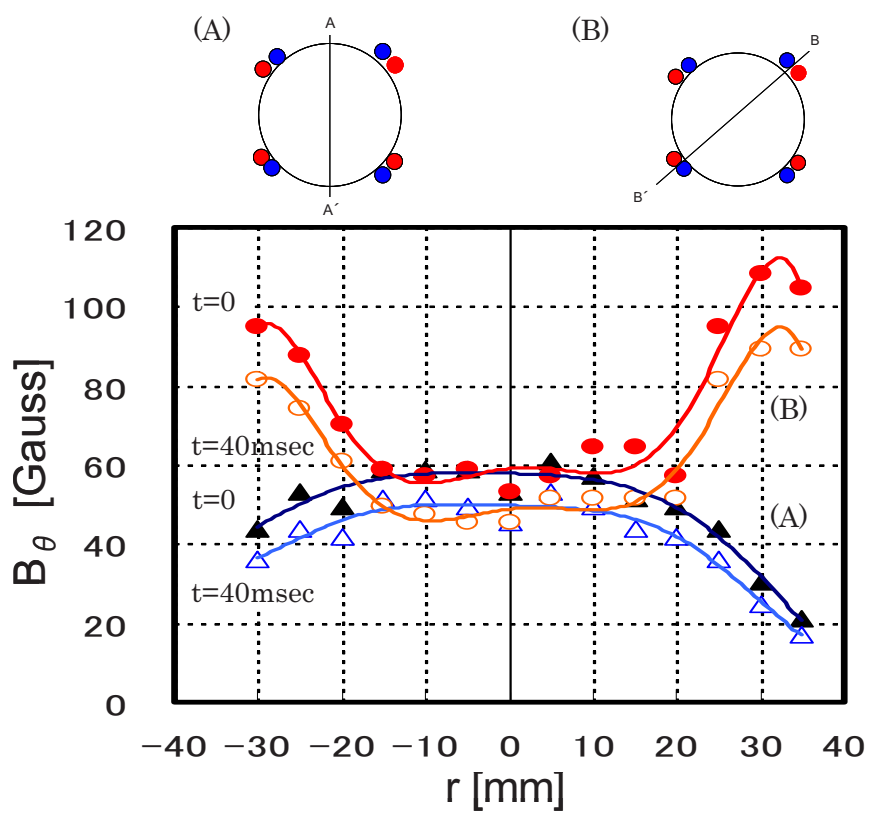

FIG. 3. (Color online) Radial dependences of the $\vartheta$ component of the RMF at $t=0$ and $t=40 \mathrm{~ms}$. The triangles and the circles show the profiles along the lines $\mathrm{A}-\mathrm{A}^{\prime}$ and $\mathrm{B}-\mathrm{B}^{\prime}$, respectively. 
(a)

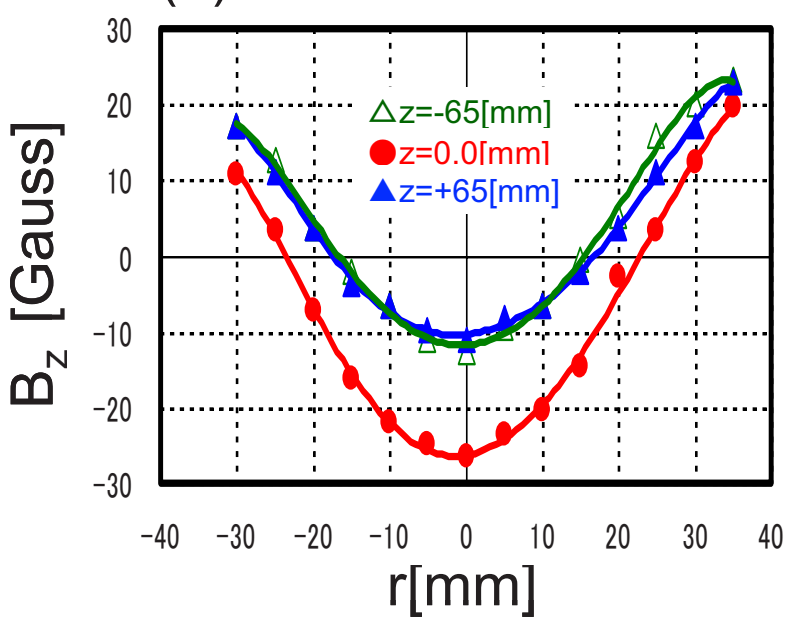

(b)

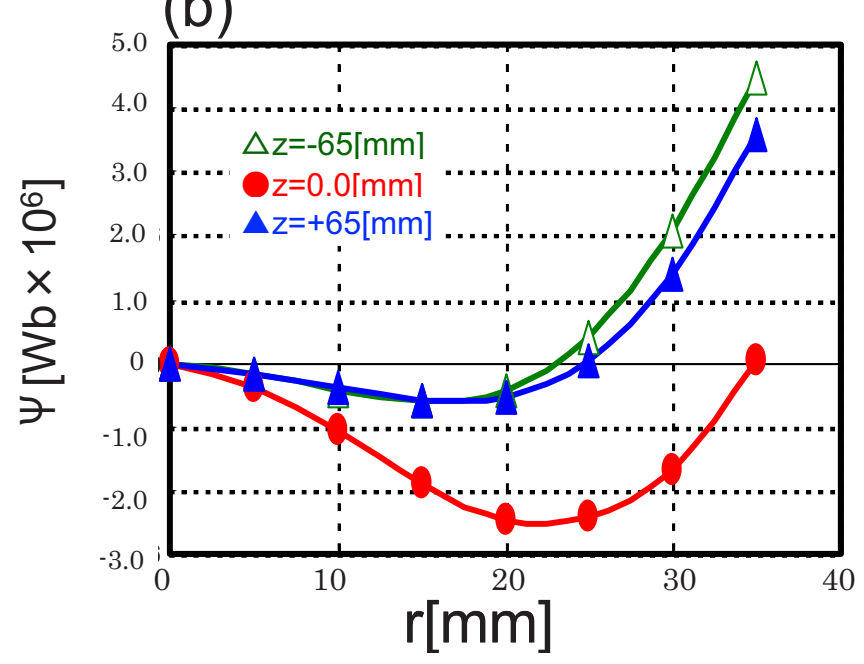

FIG. 4. (Color online) (a) Radial profiles of axial magnetic field. (b) Magnetic fluxes at the three axial positions $z=0$ and $\pm 65 \mathrm{~mm}$, respectively.

RMF continues to penetrate into the center after the formation of the FRC. The reduction of the antenna current may be caused both by an increase in the antenna resistance and by being out of the resonant condition due to the decrease of the inductance. ${ }^{14}$ As the RMF penetrates the plasma, the reduction in the inductance of the antenna becomes smaller and the antenna current increases as the resonance condition is gradually restored. The RMF gradually increases again associated with the penetration process, finally becomes about $60 \mathrm{G}$, and the RMF penetrates deeply into the plasma. The penetration conditions are satisfied throughout the discharge. Figure 3 shows the radial dependence of the $\vartheta$ component of the RMF at $t=0$ and $40 \mathrm{~ms}$ along the lines A-A' and B-B', respectively. The magnitudes of the RMF along the line A-A' are peaked at the center. On the other hand, the magnitude along the line B-B' takes a minimum value at the center and increases outward. The magnitudes of the field between at $t=0$ and $t=40 \mathrm{~ms}$ do not make a large difference.

Figures 4(a) and 4(b) show the radial profiles of the axial magnetic field and the magnetic flux evaluated from the axial field at the three axial positions $z=0$ and $\pm 65 \mathrm{~mm}$ at $t=0$, respectively. The magnetic flux shows the separatrix radius is $r=35 \mathrm{~mm}$ at $z=0$, and $r=25 \mathrm{~mm}$ at $z= \pm 65 \mathrm{~mm}$. The axial magnetic profiles of a parabolic shape indicate that the electrons are supposed to be synchronous to the RMF. The excluded magnetic flux detected by the coils wound on the glass chamber at eight positions. The signal of the coils shows that the separatrix length is about $330 \mathrm{~mm}$. The antenna length, incidentally, is $220 \mathrm{~mm}$. The plasma density and electron temperature are measured to be about $8 \mathrm{eV}$ and $2.2 \times 10^{19} \mathrm{~m}^{-3}$ at the center, respectively. The measurements are carried out by a double probe, when the FRC is in a quiet phase.

Now, the plasma pressure $p=n_{i} k T_{i}+n_{e} k T_{e} \cong n_{e} k T_{e}$ $\cong 17.6 \mathrm{~Pa}$ and the axial field pressure is $B_{z}^{2} / 2 \mu_{0} \cong 0.29 \mathrm{~Pa}$. It is then clear that the plasma pressure is not balanced by the pressure of the axial field. The RMF pressure is approximately evaluated by $B_{\theta}^{2} / 2 \mu_{0}=1 / 8 \mu_{0}\left(B_{\theta A-A}^{2},+B_{\theta B-B}^{2}\right)$ $\cong 20 \mathrm{~Pa}$, where $B_{\theta \mathrm{A}-\mathrm{A}}$, and $B_{\theta \mathrm{B}-\mathrm{B}}$, are the amplitudes of the $\theta$ component of the RMF along the lines $\mathrm{A}-\mathrm{A}^{\prime}$ and $\mathrm{B}-\mathrm{B}^{\prime}$, respectively. The pressure of the RMF is sufficient to balance the plasma pressure. We are not able to make a quantitative comparison to the theoretical analysis for two reasons. Firstly, the contribution of the RMF pressure to the pressure balance in our experiment is too large to be considered approximately as a small correction form. Secondly, we are not able to make sufficiently detailed measurements of both components of the RMF.

The authors acknowledge Dr. K. Kitano (Osaka Univ.) for his invaluable suggestion in constructing the Kansai Rotamak device.

${ }^{1}$ H. A. Blevin and P. C. Thoneman, Nucl. Fusion Suppl. 55 (1962), Part 1.

${ }^{2}$ W. N. Hugrass, I. R. Jones, and M. G. R. Philiiips, J. Plasma Phys. 26, 465 (1981).

${ }^{3}$ H. Y. Guo, A. L. Hoffman, R. D. Brooks, A. M. Peter, Z. A. Pietrzyk, S. J. Tobin, and G. R. Votroubek, Phys. Plasmas 9, 185 (2003).

${ }^{4}$ I. R. Jones, Phys. Plasmas 6, 2771 (1999).

${ }^{5}$ A. L. Hoffman, H. Y. Guo, K. E. Miller, and R. D. Milroy, Nucl. Fusion 45, 167 (2005).

${ }^{6}$ Y. Petrov, F. C. Zhong, and T. S. Huang, Phys. Plasmas 12, 082514 (2005).

${ }^{7}$ H. Y. Guo, A. L. Hoffman, and R. D. Milroy, Phys. Plasmas 14, 112502 (2007).

${ }^{8}$ W. N. Hugrass, Aust. J. Phys. 38, 157 (1985).

${ }^{9}$ R. D. Milroy, Phys. Plasmas 6, 2771 (1999).

${ }^{10}$ R. G. Storer, Plasma Phys. 24, 543 (1982).

${ }^{11}$ W. N. Hugrass, J. Plasma Phys. 28, 369 (1982).

${ }^{12}$ R. G. Storer, Nucl. Instrum. Methods 207, 135 (1983).

${ }^{13}$ L. Steinhauer, Phys. Plasmas 8, 3367 (2001).

${ }^{14}$ W. N. Hugrass, T. Okada, and M. Ohnishi, Plasma Phys. Controlled Fusion 50, 055008 (2008). 